\title{
Weakly Versus Strongly Multihop Space-Division Optical Networks
}

\author{
Alberto Bononi, Member, IEEE
}

\begin{abstract}
Transparent multihop optical networks suffer from the accumulation from node to node of crosstalk and amplified spontaneous emission noise, which may severely degrade the quality of received signals. It is thus important to keep the number of intermediate hops as low as possible. This paper compares two single-wavelength cell-switching space-division optical networks that employ deflection routing. The first has a well-known Manhattan Street (MS) distributed topology. The mean internodal distance of this network is approximately the square root of the number of nodes. We term this network as strongly multihop. The second has a centralized star topology: the star is a multistage space-division photonic switch with limited buffers. Deflected cells delivered to the wrong user are transparently rerouted to the star. This network is intrinsically single-hop and gradually becomes multihop because of deflections. We term this network as weakly multihop. As the carried traffic increases, the link load increases much more rapidly in the strongly multihop topology, and so do both the crosstalk level per hop and the number of hops caused by deflections. For the same carried traffic, the accumulated crosstalk and spontaneous emission levels in a welldesigned star-based network are much lower than in a strongly multihop network. Hence, lower packet error rates and lower delay jitter are expected for the centralized network. Moreover, for both networks, a simple frequency sweeping technique is shown to substantially reduce the dominant signal-crosstalk beat, thus allowing network operation with switch crosstalk factors as low as $-20 \mathrm{~dB}$.
\end{abstract}

Index Terms - Deflection routing, optical networks, packet switching.

\section{INTRODUCTION}

A MAJOR advantage of transparent optical networks is the possibility of flexibly upgrading transmission rates and hence network capacity by upgrading only transmitters and receivers at the access nodes, leaving the core of the network untouched. Such an advantage in management is also a major weakness in transmission. Transparency implies nonregenerative transmission from source to destination, with the ensuing degradation of the quality of signals due to accumulation of noise and distortion.

Amplified spontaneous emission (ASE) noise and deviceinduced crosstalk [1] are the two major transmission impairments in high-speed transparent optical networks in which fiber links are not long enough to introduce appreciable distortion.

Manuscript received April 16, 1997; revised December 23, 1997.

The author is with the Dipartimento di Ingegneria dell'Informazione, Università di Parma, Parma I-43100 Italy.

Publisher Item Identifier S 0733-8724(98)02960-0.
In a cell-switching environment, dynamic all-optical routing can be achieved by using deflection routing [2] with a very limited number of optical delay lines [3].

However, it is essential that cell paths be limited to a small number of hops, since each hop entails large power losses, accumulation of ASE, and crosstalk.

Thus, topologies that have mean internodal distance of at most a few hops are ideal candidates for transparent networks employing deflection routing. The usual way of decreasing the mean internodal distance is to increase the degree of each node [4]. Most practical applications of deflection routing for computer communications use indeed hypercube topologies [5], [6] with low mean internodal distance, implemented in standard electronics.

For transparent optical implementations, it is preferred to keep a small nodal degree to keep the hardware of the access node as simple as possible [7], [3], but this leads to large mean internodal distance for large network size. For instance, in the well-known Manhattan Street (MS) network [8] the mean internodal distance grows as the square root of the number of nodes [3]. We call such topologies strongly multihop.

In this paper, a centralized network $(\mathrm{CN})$ has been selected to achieve a low mean internodal distance while keeping the degree of each access node, and therefore its hardware complexity, at its bare minimum. The centralized network has a star topology, the star being a multistage space-division photonic switch with limited buffers. Deflected packets delivered to the wrong user are transparently rerouted to the star. This network is intrinsically single-hop and gradually becomes multihop because of deflections. We call this topology weakly multihop.

In this paper we compare a strongly multihop MS optical network to a weakly multihop $\mathrm{CN}$ network in terms of packet error rate (PER) for a given carried traffic (throughput).

As the carried traffic increases, the link load increases much more rapidly in the MS network, and so do both the crosstalk level per hop and the number of hops caused by deflections.

In spite of the fact that the per-hop power attenuation and crosstalk are much larger in the $\mathrm{CN}$ network, we will show that, for the same carried traffic, the crosstalk and ASE levels corrupting the cells at the receiver are much lower in a well-designed $\mathrm{CN}$ network than in the strongly multihop MS network. Moreover, the maximum carried traffic is much larger in the $\mathrm{CN}$ network, the difference becoming more and more significant for larger network size.

For on-off keying (OOK) modulation, direct detection, and a single common optical carrier frequency for all laser sources 
we find that, if the amplifier gains are suitably optimized, the dominant source of noise at the receiver is the coherent beat between signal and crosstalk.

A simple countermeasure to significantly reduce the coherent beat in such single-channel-per-fiber networks is to sweep the carrier frequency, slowly with respect to the bit rate, around its nominal value. We show that such technique substantially reduces the dominant signal-crosstalk beat, allowing network operation with switch crosstalk factors of $-20 \mathrm{~dB}$ or worse.

The paper is organized as follows. Section II introduces the network model, Section III provides the teletraffic performance in uniform traffic, Section IV derives the packet error rate for a fixed hop-length path through the network, while Section V uses the results of Sections III and IV to compute the average packet error rate, taking into account the attenuation and crosstalk characteristics of the optical switches and the ASE introduced by the optical amplifiers. Finally, Section VI summarizes the conclusions of this study.

\section{NETWORK MODEL}

The two space-division optical networks that will be compared in this paper are shown in Fig. 1.

a) The first is a two-connected distributed optical MS network with $M$ nodes. Links are unidirectional and alternate in direction like the streets and avenues in Manhattan. As shown in the figure, each node is equipped with two add-drop optical crossbar switches (A/D), an optical transmitter (TX) and an optical receiver (RX), ${ }_{1}^{1}$ and has an electronic input buffer to store incoming cells. Cells are aligned at the node by tunable optical delays (alignment stage). The header recognition block (HR) taps power off to electronically read the cell headers and make alignment/add-drop/routing decisions.

The routing block is either composed of a single crossbar (1c), or has a buffering stage composed of a second crossbar and a one-cell fiber delay loop (2c), and is driven by a shortest-path deflection routing algorithm: when a contention cannot be solved, one of the conflicting cells at random is deflected to the wrong output [3]. Deflection routing is used because en-route transparent optical buffering cannot easily be provided, since, as we will show, it introduces large power losses and crosstalk. Nodes without buffers and with a single buffer per node only will be considered, since it is known that in uniform traffic a single buffer is enough to route cells almost as efficiently as with infinitely many buffers [8].

b) The centralized network (CN) is composed of an $M \times$ $M$ space-division cell switch (active star) to which $M$ access nodes (only one is shown in the figure) are connected by dedicated fibers. Each access node is similar to the one in a), but only one optical input-output is present and only the add-drop part is provided.

The routing function is concentrated at the active star, where cells are aligned by tunable optical delays. The

\footnotetext{
${ }^{1}$ When two cells destined to the node are present simultaneously, either two optical RX's or one RX with optical buffering are required to avoid missing one of them.
}

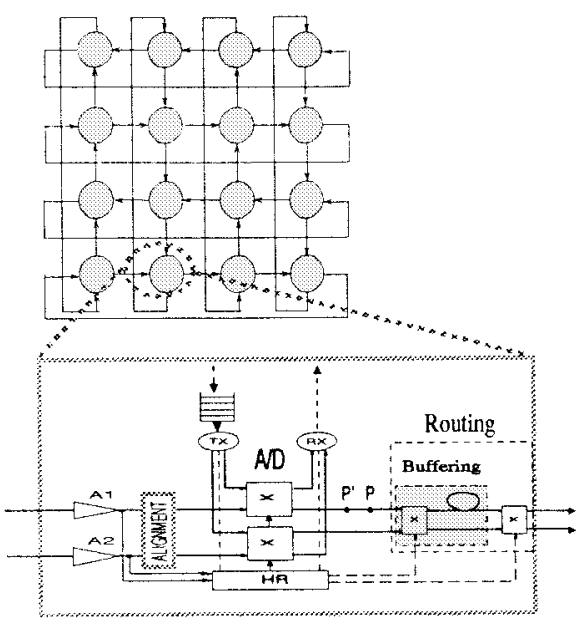

Access Node

(a)

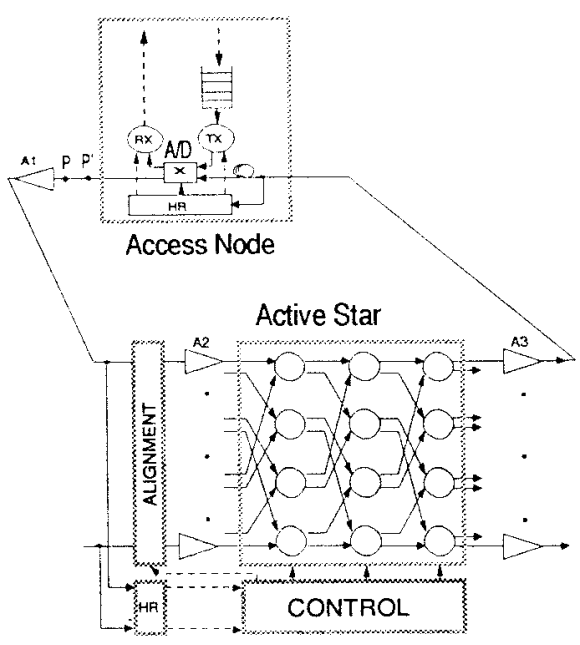

(b)

Fig. 1. (a) Distributed optical Manhattan Street network (MS), where details of the access node structure are given. (b) Centralized optical network (CN). For clarity, only one access node is drawn with details on its structure.

active star is a complete multistage photonic switch, with $\log _{2} M$ Shuffle Exchange (SX) stages based on crossbar directional couplers.

The elementary $2 \times 2$ routing elements within the active star [shown with circles in Fig. 1(b)] are either of type $1 c$ or $2 c$ like the routing block of the node in a).

Although the routing is physically centralized, the control is distributed to allow scalability at high speed. Each routing element is controlled based only on the destinations of cells at its inputs (and possibly present in its buffer). In case of contention, one cell at random is deflected to the wrong output. Deflected packets are delivered to the wrong user and transparently rerouted to the interconnect. The network, which is intrinsically single-hop, becomes gradually multihop as deflections take place because of the internal blocking of the interconnect.

\section{TELETRAFFIC}

Suppose the offered traffic is uniform, i.e., each node receives from the outside a stream of independent cells uni- 


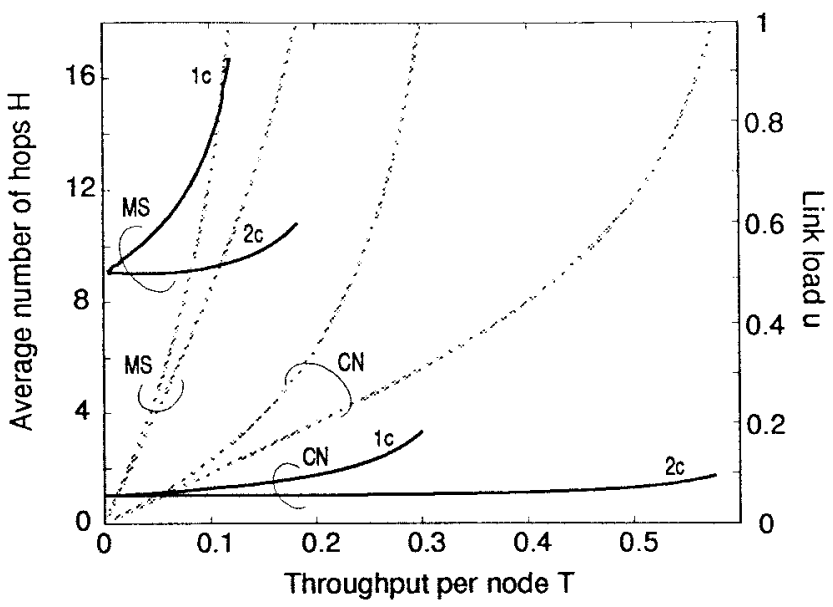

Fig. 2. Average number of hops (solid) and link load (dashed) versus throughput in uniform traffic in MS and CN with $M=256$ nodes. $1 c=$ no buffer and $2 c=$ single-buffer.

formly destined to all other nodes in the network. Let the average arrival rate at each node be $T$ cells/slot. At most one cell can be injected in the optical layer per node per slot, and cells from the input fiber(s) are given priority over local packets. As long as the input electronic buffers are not saturated, $T$ coincides with the throughput per node. Let $u$ be the link load, i.e., the probability that a slot from a link carries a cell. Given the regularity of our networks, $u$ is the same for all slots.

Appendix A shows that, if $H$ is the number of hops (i.e., crossings of access nodes) that a cell on average undergoes before reaching its destination, we get

$$
T=\frac{k u}{H}[\text { cells/slot }]
$$

where $k$ is the in-out degree of the access node, $k=1$ for $\mathrm{CN}$, and $k=2$ for MS. Note that the "mini-hops" from stage to stage inside the interconnect do not add to the hop count $H$ in (1), but of course they do add to the total crosstalk and attenuation.

For a given $u$, reducing $H$ has the positive effect of increasing the throughput $T$. The weakly multihop $\mathrm{CN}$ should then allow a much higher throughput than the strongly multihop MS. Equivalently, for the same throughput, $\mathrm{CN}$ should have a much lower slot utilization $u$, which has the positive effect of decreasing crosstalk.

Fig. 2 shows the average number of hops $H$ and the link load $u$ versus throughput in uniform traffic for $M=256$ nodes for both MS and CN. Each curve has been obtained both by simulation and by analytical models [3], [9]. For CN, simple analytical expressions are available [9].

In the MS case, the average number of hops starts from a minimum of 9.02 and quickly increases with throughput. In the $\mathrm{CN}$ case, the average number of hops starts from a minimum of one, and does not exceed 3.5 for the unbuffered (1c) case, while it is below two for the buffered $(2 c)$ case.

The strength of the centralized approach lies in the substantial decrease of the number of hops, which allows significantly lower propagation losses when the per-hop loss $L_{h}$ does not strongly depend on the number of stages, as when for instance

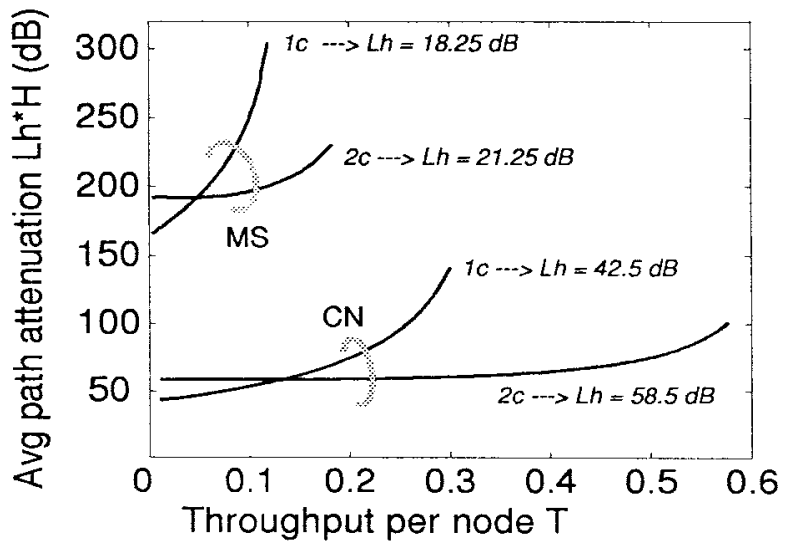

Fig. 3. Average path attenuation $L_{h} * H$ versus throughput. Values of $L_{h}$ are derived from Fig. 1(b) and Table I.

TABLE I

PaRAmeters Used in CAlculations

\begin{tabular}{c|c|c}
\hline Symbol & Meaning & Value/Range \\
\hline \hline $\mathrm{M}$ & \# of nodes & 256 \\
$\mathrm{u}$ & link load & $0-1$ \\
$\mathrm{n}$ & \# of hops & $1-1000$ \\
\hline $\mathrm{R}$ & bit rate & $1-20 \mathrm{~Gb} / \mathrm{s}$ \\
$b$ & norm. optical filter bandwidth $B_{\mathrm{o}} / R$ & 5 \\
$\Delta F / R$ & normalized sweep range & 4 \\
$\Delta_{M S}$ & node-node fiber span in MS & $5 \mathrm{~km}$ \\
$\Delta_{C N}$ & node-node fiber span in CN & $23 \mathrm{~km}$ \\
\hline$n_{s p}$ & EDFA spontaneous emission factor & 1.3 \\
$P_{s a t}$ & EDFA outpit saturation power & $10 \mathrm{dBm}$ \\
\hline$L_{f}$ & fiber loss (over $\Delta$ Km) & $0.25 * \Delta(\mathrm{dB})$ \\
$L_{h r}$ & header recognition tapping loss & $1 \mathrm{~dB}$ \\
$L_{a l}$ & alignment loss & $10 \mathrm{~dB}$ \\
$L_{a d}$ & add/drop coupler loss & $3 \mathrm{~dB}$ \\
\hline$x$ & \# of crossbars in routing element & 1 for $1 c, 2 \mathrm{for} 2 c$ \\
$L_{r}$ & routing block loss in MS & $3 * x(\mathrm{~dB})$ \\
\hline $\mathrm{s}$ & $\#$ of couplers crossed at the interconnect & $x * \log \cdot \mathrm{M}$ \\
$L_{i c}$ & interconnect loss (partial integration) & $2 * \mathrm{~d}(\mathrm{~dB})$ \\
\hline$\alpha$ & switch crosstalk factor & $-23 \mathrm{~dB}$ \\
$N_{x}$ & \# of crosstalk points & $n * x+2$ for $\mathrm{MS}$ \\
& & $n * s+2$ for CN \\
\hline$P_{t x}$ & transmitted mark power & $0 \mathrm{dBm}$ in CN \\
\hline$N_{b}$ & \# bits/cell & 1000 \\
\hline
\end{tabular}

alignment and fiber attenuation dominate. Fig. 3 shows the average path attenuation $L_{h} * H$ versus throughput. The values of $L_{h}$ are calculated from Fig. 1(b) and Table I. The per-stage loss in $\mathrm{CN}$ has been assumed $2 \mathrm{~dB}$ for $1 c$ and $4 \mathrm{~dB}$ for $2 c$ assuming a hybrid integration. Since clearly $\mathrm{CN}$ has lower path attenuation, with a careful choice of amplifier gains it should also have lower ASE levels.

Fig. 2 also shows the increase of the fraction of nonempty slots $u$ with throughput, which causes an increase of the accumulated crosstalk. For the same carried traffic $T$, it is seen that $u$ is much lower in CN than in MS. As we will see, this has important implications on the PER curves.

\section{TRANSMISSION}

This section will derive the bit error rate $\operatorname{BER}(n)$ in a tagged cell that has hopped $n$ times before reaching its destination. All symbols of interest are given in Table I, along with the values used in the analysis. 
All transmitter lasers have a common nominal optical frequency $\nu_{0}=\omega_{0} / 2 \pi$, and are externally OOK modulated with nonreturn-to-zero pulses at a bit rate $R=1 / T$ where $T$ is the bit time. All TX's have the same power $P_{t x}$ on mark and zero on space.

The direct-detection receiver consists of a bandpass optical filter of bandwidth $B_{O}$ centered at the carrier frequency $\nu_{0}$, a polarization filter, a photodiode, and a matched (integrateand-dump) filter, followed by a sampler and by the decision circuitry [10]. The presence of the polarization filter is only for analytical simplicity and does not appreciably affect the results [10].

We fix the attention on a tagged bit of a cell taking $n$ hops and reaching its intended receiver. It collects crosstalk at the routing switches and ASE noise in the optical amplifiers. The objective is to find the probability of error on such a bit.

\section{A. Amplification: Distributed Network}

Assume that nodes are regularly spaced $\Delta_{\mathrm{MS}} \mathrm{km}$ apart on a square grid. Two doped-fiber amplifiers $A_{1}, A_{2}$ are placed at the inputs of the node to compensate for fiber and node attenuation. We assume the amplifiers have power gain $G$, constant for all input powers up to saturation, and output saturation power $P_{\text {sat }}$. The ASE added at the output of each amplifier is an additive white Gaussian noise with (one-sided) power spectral density $h \nu_{0} n_{s p}(G-1)^{2}$ where $h$ is Planck's constant and $n_{s p}$ is the spontaneous emission factor.

To keep equal power levels in the network, the amplifiers are set to exactly compensate the per-hop loss

$$
G=L_{h r} L_{a l} L_{a d} L_{r} L_{f}
$$

where the losses are defined in Table I.

With the aid of Fig. 1(a), the ASE power density accumulated by the test bit in one hop from point $\mathrm{P}$ to point $\mathrm{P}$ ' of the next node is

$$
N_{\text {ase }}(1)=h \nu n_{s p}(G-1) /\left(L_{h r} L_{a l} L_{a d}\right) .
$$

From Table I, we get $N_{\text {ase }}(1)=4.4 \times 10^{-19} \mathrm{~W} / \mathrm{Hz}$ for $1 c$ and $N_{\text {ase }}(1)=8.8 \times 10^{-19} \mathrm{~W} / \mathrm{Hz}$ for $2 c$. Given constraint (2), such noise contribution remains constant until absorption, and each hop contributes the same noise level, so that at the receiver $N_{\text {ase }}(n)=n N_{\text {ase }}(1)$. Optical filters of bandwidth $B_{o}$ follow each amplifier to avoid saturation due to ASE.

Since the power of cells coming from the network at the A/D switch of the access node cannot exceed $P_{\text {sat }} / L_{a l} L_{h r}$, if newly injected packets are desired to have the same power level as hopping packets, then the transmitted power should satisfy

$$
P_{t x} L_{a l} L_{h r} \leq P_{\text {sat }}
$$

Equality can be chosen to maximize the optical signal-to-noise ratio (SNR). Note that changing the location of amplifiers, for instance placing them at the output, does not change the maximum SNR, but only the required transmitter power.

\footnotetext{
${ }^{2}$ Along the polarization direction of the signal.
}

\section{B. Amplification: Centralized Network}

If the nodes are regularly spaced $\Delta_{\mathrm{MS}} \mathrm{km}$ apart on a square grid, and the star is placed at the center of the square, the average fiber link length from node to star is approximately [11] $\Delta_{\mathrm{CN}} \cong 0.28 \sqrt{M} \Delta_{\mathrm{MS}}$. To simplify the following analysis, all links are assumed to have the same length $\Delta_{\mathrm{CN}}$.

Doped-fiber amplifiers $A_{1}, A_{2}, A_{3}$ with output saturation power $P_{\text {sat }}$ are placed as in Fig. 1(b). To keep equal power levels in the network, the amplifiers are set to have unity round-trip gain

$$
G_{1} G_{2} G_{3}=L_{1} L_{2} L_{3}
$$

where the interamplifier losses are $L_{1}=L_{f} * L_{h r} * L_{a l}, L_{2}=$ $L_{i c}, L_{3}=L_{f} * L_{n}$, the symbols being defined in Table I.

The unity gain constraint (3) allows a drastic simplification in the optimization of the PER. With this constraint, all cells that meet at a crossbar have the same power level, and thus all crosstalk terms are independent of the amplifier gains, as we will see in (6). Such gains can thus be optimized to minimize the impact of ASE, without worrying about crosstalk. As we will see in (18), the main contribution of ASE to the PER is the beat between signal and ASE which is minimized by maximizing the optical SNR, i.e., by minimizing the ratio $N_{\text {ase }}(1) / P_{r x}$.

The position and the gain of the amplifiers have been selected according to the optimization in Appendix B. Amplifier (A.1) acts as a booster, with gain $G_{1}=P_{\text {sat }} / P_{r x}$, being $P_{r x}$ the power at point $\mathrm{P}$ in Fig. 1(b). Amplifier (A.2) has gain $G_{2}=L_{1}$, bringing the output power back to the saturation value. Finally, (A.3) is chosen to satisfy (3). The key optimization concept is that the optical SNR is maximized by having the amplifiers work at the edge of saturation (gain selection criterion), and by breaking up the lumped losses between amplifiers so as to balance them as much as possible (amplifier placement criterion).

With the aid of Fig. 1(b), the ASE power density accumulated by the test bit in one hop from point $\mathrm{P}$ to $\mathrm{P}^{\prime}$ is seen to be

$$
\begin{aligned}
N_{\text {ase }}(1)= & h \nu n_{s p} \\
& \cdot\left[\frac{\left(G_{1}-1\right) G_{2} G_{3}}{L_{1} L_{2} L_{3}}+\frac{\left(G_{2}-1\right) G_{3}}{L_{2} L_{3}}+\frac{G_{3}-1}{L_{3}}\right]
\end{aligned}
$$

since the ASE processes are independent. As before, given constraint (3), $N_{\text {ase }}(n)=n N_{\text {ase }}(1)$.

For the values in Table $\mathrm{I}$, we get that for $1 c$ the gains are $\left(G_{1}, G_{2}, G_{3}\right)=(13,16.75,12.75)(\mathrm{dB})$, and $N_{\text {ase }}(1)=$ $8.54 \times 10^{-19}(\mathrm{~W} / \mathrm{Hz})$; for $2 c$ the gains are $\left(G_{1}, G_{2}, G_{3}\right)=$ $(13,16.75,28.75)(\mathrm{dB})$, and $N_{\text {ase }}(1)=1.37 \times 10^{-17}(\mathrm{~W} / \mathrm{Hz})$.

\section{Photodetection}

As the tagged cell hops from node to node, it collects crosstalk from the optical fields simultaneously crossing the same crossbar switches, and ASE noise at the amplifiers.

When it reaches its intended receiver after $n$ hops, the complex envelope (with respect to the nominal frequency $\nu_{0}$ ) 
of the optical field at the photodiode is $\tilde{e}_{r x}=\tilde{e}_{0}+\tilde{e}_{x t}+\tilde{e}_{\text {ase }}$ where

$$
\begin{aligned}
& \tilde{e}_{0} \\
& \tilde{e}_{x t} \\
& \tilde{e}_{\text {ase }}
\end{aligned}
$$$$
\tilde{e}_{x t}=\sum_{i=1}^{n_{x t}} \tilde{e}_{x t}(i) \text { the accumulation of } n_{x t} \text { crosstalk in- }
$$
terferers; the complex envelope of the accumulated ASE noise, which is an additive Gaussian bandpass process of flat onesided spectral density $N_{\text {ase }}(n)$ over the optical filter bandwidth $B_{0}$ centered around $\nu_{0}$.

The desired signal during the tagged bit time $(0<t<T)$ is

$$
\tilde{e}_{0}=\sqrt{2 P_{r x}} m_{0} e^{j\left(\delta \omega_{0} t+\phi_{0}\right)} \quad(\sqrt{\mathrm{W}})
$$

where

$P_{r x} \quad$ the received mark power;

$m_{0}=1$ for mark, and zero for space;

$\phi_{0} \quad$ a random variable (RV), uniform over $[-\pi, \pi]$ and constant over the bit time $T$, accounting for phase noise of the TX laser and other possible sources of phase uncertainty; assuming $\phi_{0}$ constant over the bit time amounts to assuming that the TX laser linewidth is significantly smaller than the bit rate $R$;

$\delta \omega_{0} \quad$ a random frequency offset from the nominal carrier $\omega_{0}$, uniform over $[-\pi \Delta F, \pi \Delta F]$. Such offset models a slow frequency drift of the TX laser, which could be obtained by modulating the laser current by a sawtooth sweep signal or simply by loosely stabilizing the thermal drift of the laser. We impose $\Delta F<B_{0}-R$ to prevent the signal from drifting off the optical filter.

Each crosstalk interferer can be expressed as

$$
\tilde{e}_{x t}(i)=\sqrt{2 \alpha P_{r x}} m_{i}(t) \cos \Psi_{i} e^{j\left(\delta \omega_{i} t+\phi_{i}\right)} \quad(\sqrt{\mathrm{W}})
$$

where

$\delta \omega_{i}$ and $\phi_{i} \quad$ independent, identically distributed (i.i.d.) RV's, with the same statistics as $\delta \omega_{0}$ and $\phi_{0}$, respectively;

$m_{i}(t) \in\{0,1\}$ the OOK modulation of the interferers, and the dependency on time accounts for the random bit-misalignment of the various OOK channels;

$\alpha \quad$ the power crosstalk factor of each crossbar. Since $\alpha \ll 1$, only first-order crosstalk factors are considered, i.e., terms whose power is proportional to $\alpha^{j}, j>1$, are neglected. All crosstalk interferers have the same power, since conditions (2) and (3) impose that cells that meet at a crossbar have the same power level, no matter how many hops they have taken ${ }^{3}$;

$\cos \Psi_{i}$ the polarization component of the $i$ th interferer along the polarization direction

\footnotetext{
${ }^{3}$ This is true if the extra power due to accumulation of crosstalk and ASE on a cell can be neglected
}

of the tagged cell. The angles $\Psi_{i}$ are assumed to be independent RV's, uniform over $[-\pi, \pi]$. Although a practical receiver will not keep track of the desired signal's polarization state, neglecting orthogonal (noncoherent) components has little impact on performance, since the crucial crosstalk contribution is the coherent beat with the signal. Also, to avoid worst case beating effects, especially in nonuniform traffic, it is useful that the transmitters change randomly their polarization at each transmitted cell.

The input field can be rewritten as

$$
\tilde{e}_{r x}=\sqrt{2 P_{r x}} e^{j\left(\delta \omega_{0} t+\phi_{0}\right)}\left[m_{0}+\tilde{x}(t)+\tilde{a}(t)\right]
$$

where the normalized crosstalk field is

$$
\tilde{x}=x_{i}(t)+j x_{q}(t)=\sqrt{\alpha} \sum_{i=1}^{n_{x t}} m_{i}(t) \cos \Psi_{i} e^{j\left(\Delta \omega_{i} t+\Phi_{i}\right)}
$$

where $\Delta \omega_{i}=\delta \omega_{i}-\delta \omega_{0}$ and $\Phi_{i}=\phi_{i}-\phi_{0}$.

The normalized ASE field is

$$
\tilde{a}(t)=a_{i}(t)+j a_{q}(t)=\frac{\tilde{e}_{\text {ase }}(t)}{\sqrt{2 P_{r x}}} e^{-j\left(\delta \omega_{0} t+\phi_{0}\right)} .
$$

The current after the photodetector is

$$
i(t)=I_{p}(t)+i_{s n}(t)+i_{t h}(t)
$$

where $I_{p}(t)=(\mathcal{R} / 2)\left|\tilde{e}_{r x}(t)\right|^{2}, \mathcal{R}$ is the responsivity of the photodetector $(\mathrm{A} / \mathrm{W})$. Since the received power is large, we neglect both the shot noise current and the thermal noise of the electronic circuitry.

During a mark signal bit $I_{p}$ can be written as

$$
I_{p}=\mathcal{R} P_{r x}\left\{1+n_{s^{-x t}}+n_{s^{-} s p}+n_{x t-x t}+n_{x t^{-s p}}+n_{s p-s p}\right\}
$$

where the contributions of the beat terms between signal and crosstalk $(s-x t)$, signal and ASE $(s-s p)$, crosstalk with itself $(x t-x t)$, crosstalk with ASE $(x t-s p)$, and ASE with itself $(s p-s p)$ are

$$
\begin{aligned}
n_{s^{-} x t}(t) & =2 x_{i}(t) \\
n_{s^{-} s p}(t) & =2 a_{i}(t) \\
n_{x t^{-} s p}(t) & =2\left[x_{i}(t) a_{i}(t)+x_{q}(t) a_{q}(t)\right] \\
n_{x t^{-} x t}(t) & =x_{i}^{2}(t)+x_{q}^{2}(t) \\
n_{s p-s p}(t) & =a_{i}^{2}(t)+a_{q}^{2}(t) .
\end{aligned}
$$

The dominant terms are the coherent beats with the signal $n_{s^{-s p}}$ and $n_{s^{-} x t}$. The term $n_{x t^{-x t}}(t)$ is always much smaller than $n_{s^{-} x t}(t)$ and is thus neglected for $\alpha \ll 1 .^{4}$ The term $n_{s p-s p}(t)$ becomes significant with respect to $n_{s-s p}(t)$ only when the optical filter bandwidth $B_{\circ}$ is very large [12], and thus must be included in the analysis of the frequency sweeping technique. Finally, the term $n_{x t^{-} s p}(t)$ is always much smaller than $n_{s-s p}(t)$ and is neglected.

\footnotetext{
${ }^{4}$ It has variance proportional to $\alpha^{2}$, like the coherent beats between secondorder crosstalk terms and signal. Consistently with keeping track of only first-order crosstalk, such term should be neglected.
} 
The normalized statistic at the decision gate can then be expressed as

$$
Z=\left(\frac{1}{\mathcal{R} P_{r x}}\right) \frac{1}{T} \int_{0}^{T} i(t) d t=1+Y_{s^{-x t}}+Y_{s^{-} s p}+Y_{s p-s p}
$$

where $Y_{\bullet}=\frac{1}{T} \int_{0}^{T} n_{\bullet}(t) d t$.

As the number of i.i.d. crosstalk interferers $n_{x t}$ gets large, the distribution of $Y_{s-x t}$ tends, by the central limit theorem, to a Gaussian RV. Hence, the BER can be estimated by the usual Gaussian approximation [12], [13]. It has been shown that, in the presence of crosstalk, the Gaussian approximation overestimates the BER [14].

Using Personick's formula, we get

$$
\operatorname{BER}(n)=Q\left(\frac{1}{\sqrt{\sigma_{s-x t}^{2}+\sigma_{s-s p}^{2}+\sigma_{s p-s p}^{2}}+\sqrt{\sigma_{s p-s p}^{2}}}\right)
$$

where $Q(x)=(1 / \sqrt{2 \pi}) \int_{x}^{\infty} e^{-y^{2} / 2} d y$, and $\sigma_{0}^{2}$ denotes variance of $Y_{0}$.

1) Signal-Crosstalk Beat: Appendix $C$ shows that $x_{i}(t)$ and $x_{q}(t)$ are uncorrelated and have the same autocorrelation

$$
\begin{aligned}
R_{x_{i}}(\tau) & =\mathrm{E}\left[x_{i}(t) x_{i}(t+\tau)\right] \\
& =\frac{\alpha \mathrm{E}\left[n_{x t}\right]}{4} \operatorname{sinc}^{2}(\Delta F \tau) R_{m}(\tau)
\end{aligned}
$$

where the average number of crosstalk terms $\mathrm{E}\left[n_{x t}\right]$ is also derived in the appendix. The autocorrelation of the OOK asynchronous modulation signals $m_{i}(t)$ is [15] $R_{m}(\tau)=$ $\frac{1}{4}(1+\Lambda(\tau / T)) .{ }^{5}$ For bit-aligned interferers we have $R_{m}(\tau)=$ $1 / 2$.

The variance of $Y_{s^{-} x t}$ is calculated as $\sigma_{s^{-} x t}^{2}=$ $\left(4 m_{0} / T^{2}\right) \int_{0}^{T} \int_{0}^{T} R_{x_{i}}(\beta-\alpha) d \alpha d \beta$. A closed form of this double integral can be found, giving

$$
\sigma_{s^{-x t}}^{2}=\frac{\mathrm{E}\left[n_{x t}\right]}{2} \alpha \eta(\rho)
$$

where $\rho=2 \pi \Delta F / R$, and the beat efficiency factor $\eta(\rho)$ is shown in (17) at the bottom of the page where $\gamma_{e}=0.5772 \cdots$ is euler's gamma, and $\operatorname{Si}(\rho)$ and $\operatorname{Ci}(\rho)$ are the sine and cosine integrals, respectively [17]. The top expression is for bit-asynchronous interferers, the bottom one for bit-aligned (synchronous) interferers. For large $\rho$, in both cases we have $\eta(\rho) \cong 2 \pi / \rho$.

Fig. 4 shows $\eta$ versus the normalized sweep range $\Delta F / R$. It is seen that both the bit-aligned and the asynchronous curves quickly converge to $R / \Delta F$. For the asynchronous case, $\eta(0)=5 / 6$. From (16) one can see that one can trade $\Delta \alpha \mathrm{dB}$ 's

${ }^{5}$ The triangular function is defined as $\Lambda(t / x)=1-|t| / x$ for $|t|<x$ and zero outside.

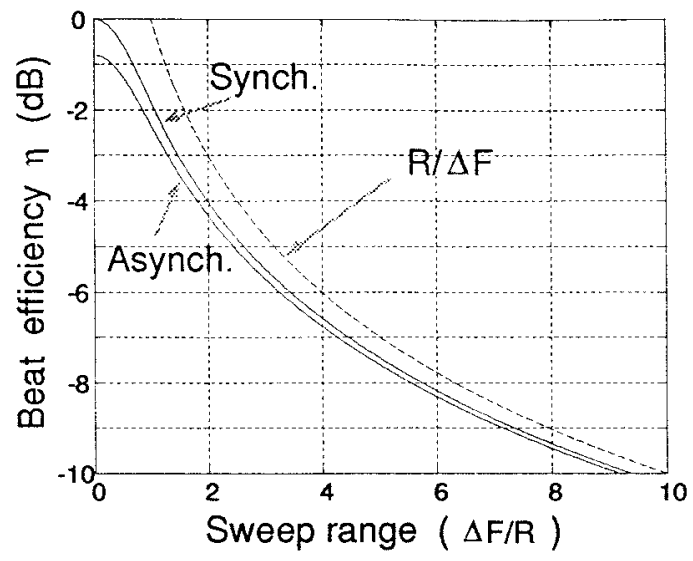

Fig. 4. Beat efficiency $\eta$ versus normalized sweep range $\Delta F / R$.

in the switch crosstalk factor for $\Delta \eta \mathrm{dBs}$ in the beat efficiency by acting on the sweep range $\Delta F / R$.

2) Signal-ASE and ASE-ASE Beat: From (9), we can rewrite $\tilde{a}(t)=\tilde{n}(t) e^{-j \delta \omega_{0} t}$, where $\tilde{n}=n_{i}+j n_{q}$ is a lowpass zero-mean complex Gaussian process, and where $n_{i}$ and $n_{q}$ are uncorrelated, with the same spectral density ${ }^{6}$ $S_{n_{i}}(f)=\left(N_{\text {ase }}(n) / 2 P_{r x}\right) \Pi\left(f / B_{o}\right)$.

The term $w(t)=e^{-j \delta \omega_{0} t}$ is recognized as a frequency modulation, so that we get [16] $R_{w}(\tau)=\mathrm{E}\{w(\tau)\}=\operatorname{sinc}(\Delta F \tau)$ and therefore the spectrum $S_{w}(f)=(1 / \Delta F) \Pi(f / \Delta F)$.

Since the probability density of $\delta \omega_{0}$ is symmetric around zero, one finds that the real and imaginary part of $\tilde{a}, a_{i}$ and $a_{q}$, are also uncorrelated and have identical spectrum

$$
S_{a_{i}}(f)=S_{n_{i}}(f) \otimes S_{w}(f)
$$

where $\otimes$ indicates convolution. Such convolution has value $\left(N_{\text {ase }}(n) / 2 P_{r x}\right)$ for $|f|<\left(B_{O}-\Delta F\right) / 2$, and linearly decreases to zero for $\left(B_{o}-\Delta F\right) / 2<|f|<\left(B_{o}+\Delta F\right) / 2$.

Now, $a_{i}(t)$ is filtered by the integrator of noise bandwidth $R / 2$. Since we imposed $B_{o}-\Delta F \geq R$, filtering $a_{i}(t)$ is essentially the same as filtering $n_{i}(t)$ only, i.e., the multiplying exponential term in (9) has negligible effect on the variance of the term $n_{s^{-} s p}$, which simply is

$$
\sigma_{s^{-} s p}^{2} \cong 2 \frac{N_{\text {ase }}(1)}{P_{r x}} n R \text {. }
$$

Evaluation of the term $\sigma_{s p-s p}^{2}$ is more involved. However, without frequency sweeping we have the well-known result [12], [18]

$$
\sigma_{s p-s p}^{2} \cong(4 b-1)\left(\frac{\sigma_{s-s p}^{2}}{4}\right)^{2}
$$

${ }^{6}$ The rectangular function is defined as $\Pi(t / x)=1$ for $|t|<x / 2$ and zero outside.

$$
\eta(\rho)= \begin{cases}\frac{2}{\rho^{2}}\left(-3 \gamma_{e}-3 \ln (\rho)+3 \operatorname{Ci}(\rho)+2 \rho \operatorname{Si}(\rho)-1+2 \cos (\rho)-\operatorname{sinc}(\rho / \pi)\right) & \text { asynch. } \\ \frac{4}{\rho^{2}}\left(-\gamma_{e}-\ln (\rho)+\operatorname{Ci}(\rho)+\rho \operatorname{Si}(\rho)-1+\cos (\rho)\right) & \text { synch. }\end{cases}
$$




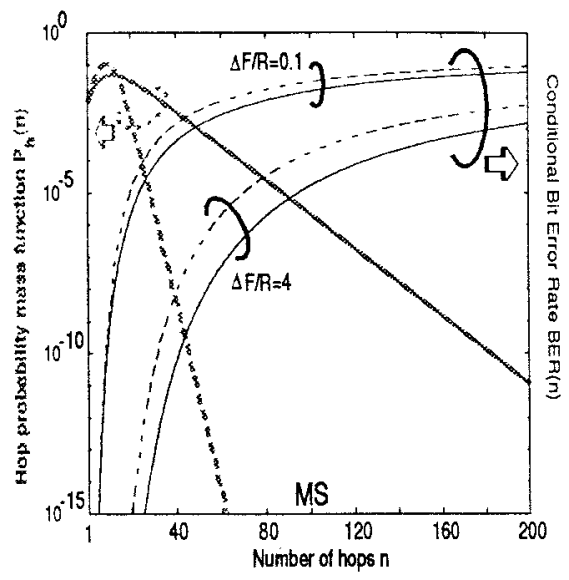

(a)

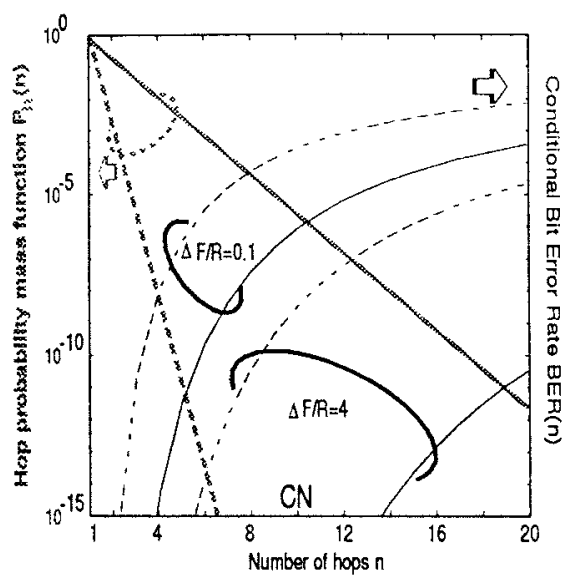

(b)

Fig. 5. $\operatorname{BER}(n)$ and hop distribution $p_{h}(n)$ versus hop number $n$ in (a) MS and (b) $\mathrm{CN}$ for a fixed throughput $T=0.12$ and bit rate $R=20 \mathrm{~Gb} / \mathrm{s}$. Solid: no buffer $1 c$; Dotted: single buffer $2 c$. Note that the hop scale for $\mathrm{CN}$ is ten times smaller than for MS.

Sweeping broadens the spectrum of $n_{s p^{-} s p}(t)$, so that the variance is actually lower than (19) because less energy passes through the integrator. It can be shown that for large optical filter $(b \gg 1)$ and broad sweep range $(\Delta F / R \cong b)$ the expression in (19) should be multiplied by two-thirds.

\section{COMBINING TeletrafFIC AND TRANSMission}

The system parameters used in this results section are given in Table I, unless otherwise noted. No polarization dependency is assumed for gains and losses.

Assuming cells of $N_{b}$ bits and errors independent bit by bit, the unconditional packet error rate is obtained by conditioning on the number of hops $n$ taken by a typical cell in the network as

$$
\mathrm{PER}=\sum_{n=1}^{\infty}\left[1-(1-\operatorname{BER}(n))^{N_{b}}\right] p_{h}(n)
$$

where $p_{h}(n)$ is the probability mass function of the number of hops $h$ [3], [9]. Since noises are not uncorrelated bit-by-bit, (20) is indeed an upper-bound.

Fig. 5 shows the two key components of (20), namely, conditional bit error rate $\operatorname{BER}(n)$ and hop-distribution $p_{h}(n)$.

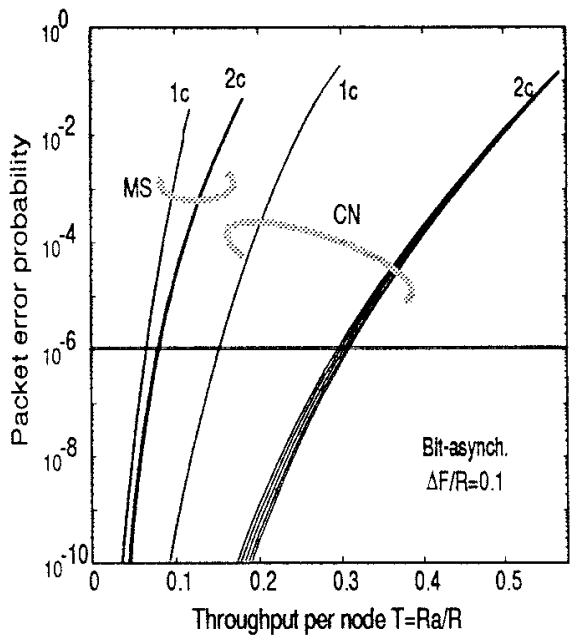

(a)

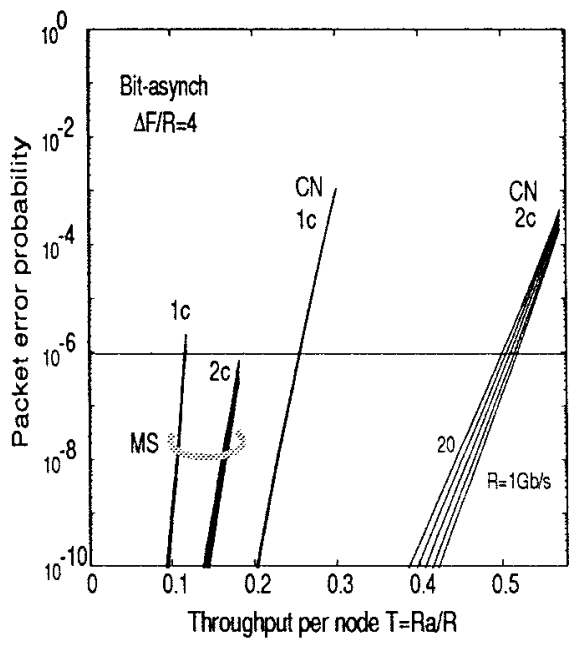

(b)

Fig. 6. PER versus throughput in MS and $\mathrm{CN}$ for (a) low sweep $(\Delta F / R=0.1)$ and (b) substantial sweep $(\Delta F / R=4)$. Curves are given for bit rate $R=1,5,10,15$, and $20 \mathrm{~Gb} / \mathrm{s}$.

Dotted lines refer to $2 c$ elements, and solid lines to $1 c$ elements. The curves are for (a) MS and (b) $\mathrm{CN}$, for a fixed throughput $T=0.12$ and bit rate $R=20 \mathrm{~Gb} / \mathrm{s}$. As seen in Fig. 2, this is the maximum throughput $T$ achievable with MS-1 $c$ at full load $(u=0.99)$. At this value of $T$, MS- $2 c$ has $u=0.56, \mathrm{CN}-1 c$ has $u=0.16$, and $\mathrm{CN}-2 s$ has $u=0.12$. Such lower loads allow lower values of crosstalk.

Interestingly, the slope of the hop-distribution curve is almost ten times steeper in $\mathrm{CN}$, due to its weakly multihop nature. However, since the per-hop power losses (and hence the ASE due to their compensation by optical amplification) are much larger in $\mathrm{CN}$, the $\operatorname{BER}(n)$ curves grow much faster with $n$. Sweeping the optical carrier across almost all of the optical filter bandwidth $\left(B_{0}=5 R\right)$ using $\Delta F / R=4$ is beneficial in all cases where signal-crosstalk beat dominates.

A meaningful way of comparing different topologies for the same carried traffic is to give PER versus throughput curves. These are shown in Fig. 6, with bit rate $R$ as a parameter, ranging from 1 to $20 \mathrm{~Gb} / \mathrm{s}$. Note that, for a given transmitter 


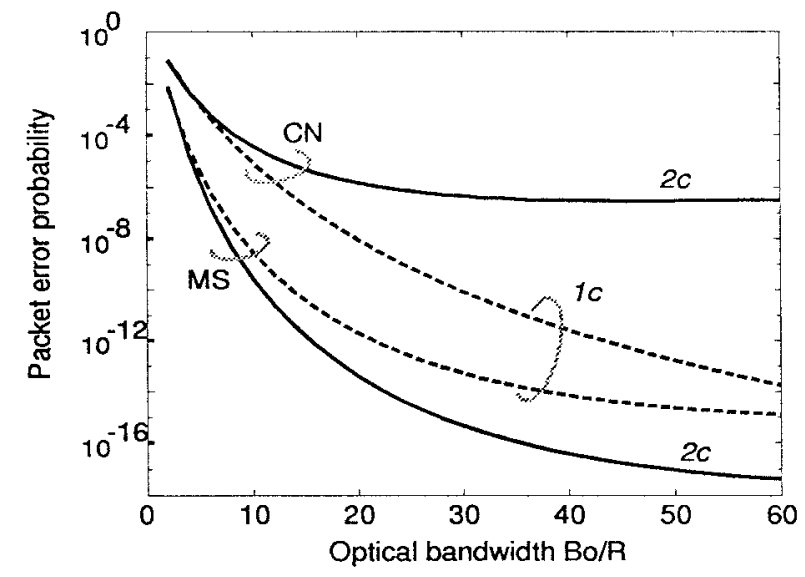

Fig. 7. PER versus normalized optical bandwidth $b=B_{o} / R$ when all slots are full $(u=1)$ for $R=20 \mathrm{~Gb} / \mathrm{s}$. The normalized sweep range is $\Delta F / R=b-1$.

bit rate $R$, the abscissa can be read as the actual sustained rate $R_{a}$ at the access node, since $T=R_{a} / R$.

In Fig. 6(a), there is essentially no frequency sweeping $\Delta F / R=0.1$ and all curves depend very weakly on $R$. This indicates that, given the poor crosstalk factor $\alpha=-23 \mathrm{~dB}$, coherent crosstalk is the dominant impairment since, as seen from (16), it is bit rate independent. Therefore, they should greatly improve with sweeping.

In Fig. 6(b), there is sweeping all across the optical filter bandwidth, $\Delta F / R=4$, which gives a beat efficiency of $\eta \cong 7$ $\mathrm{dB}$. Thus the effective crosstalk factor is $\eta \alpha \cong-30 \mathrm{~dB}$ and PER substantially improves. The fan of $\mathrm{CN}-2 c$ curves with $R$ as a parameter starts to open up. This bit rate dependency shows that signal-spontaneous beat starts to be the dominant factor. A larger sweep range (allowed by a larger optical filter) should not push the PER curves of $\mathrm{CN}-2 c$ down the ordinates axis as much as it would do for $\mathrm{MS}$ and $\mathrm{CN}-1 c$, in which coherent crosstalk beat still dominates.

The effect of enlarging the sweep range is shown Fig. 7 where the decrease of the full-load (top) points of the PER curves in Fig. 6 is shown versus the normalized optical bandwidth $b=B_{o} / R$, assuming a maximum sweep range $\Delta F / R=(b-1)$. The PER decreases as the coherent crosstalk beat decreases, it flattens out when the signalspontaneous beat dominates, and then starts increasing again when the spontaneous-spontaneous beat becomes dominant because of the very large optical bandwidth (this last increase is not shown in the figure). The range of values at which the PER is minimum is very broad, as can be seen for the $\mathrm{CN}-2 c$ case, which displays a floor right below $10^{-6}$ for optical bandwidth $b$ above 20. As already inferred from Fig. 6, the other networks, MS and $\mathrm{CN}-1 c$, need larger optical bandwidths to reach their minimum, and benefit mostly from the sweeping technique. Note however that Fig. 7 shows PER curves for the same link load (full-load) and this does not give a fair comparison among topologies, which should instead be compared for the same carried traffic.

It is worth to point out the great sensitivity of the $\mathrm{CN}$ network to the amplifier placement. If for instance we move the (A.2) amplifiers of Fig. 1(b) to the left of the alignment

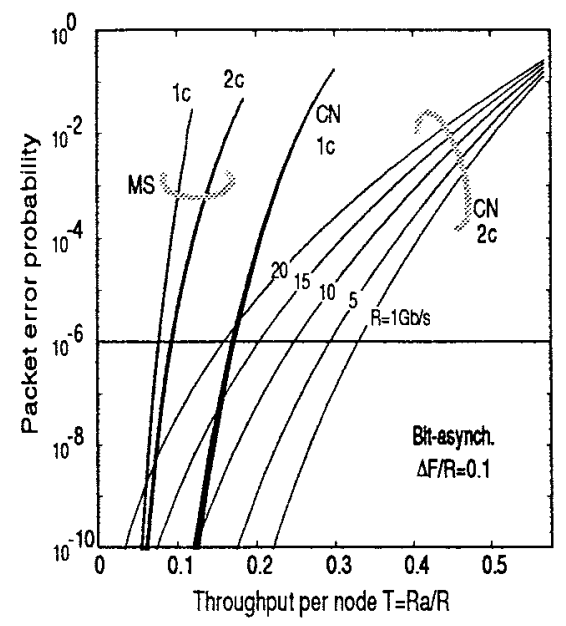

(a)

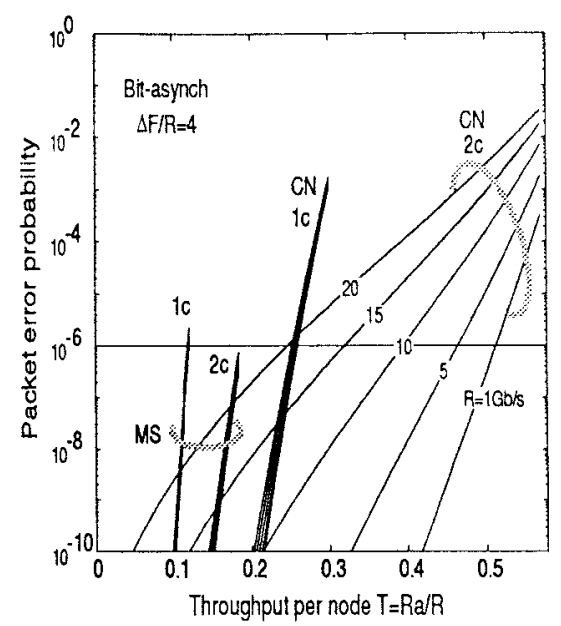

(b)

Fig. 8. PER versus throughput for the nonoptimal placement of EDFA's in $\mathrm{CN}$ for (a) low sweep $(\Delta F / R=0.1)$ and (b) substantial sweep $(\Delta F / R=4)$. MS curves are also given for comparison.

stages, a very large lumped loss at the interconnect results. For this placement, from Appendix B and the values in Table I we get that for $1 c$ the optimal gains are $\left(G_{1}, G_{2}, G_{3}\right)=$ $(13,6.75,22.75)(\mathrm{dB})$, and $N_{\text {ase }}(1)=3.5 \times 10^{-18}(\mathrm{~W} / \mathrm{Hz})$; for $2 c$ the optimal gains are $\left(G_{1}, G_{2}, G_{3}\right)=(13,6.75,38.75)$ $(\mathrm{dB})$, and $N_{\text {ase }}(1)=1.31 \times 10^{-16}(\mathrm{~W} / \mathrm{Hz})$. The curves of Fig. 8 result. These should be compared to those of Fig. 6 . We note that in this case the curves display a much stronger sensitivity to the TX bit rate, since now the signal-spontaneous beat is dominant, and much worse PER results. The best network performance would thus be obtained by breaking the losses as much as possible, as for instance in a realization of the interconnect with semiconductor optical amplifier (SOA) crossbars [19], instead of the lumped erbium doped amplifiers (EDFA) we have assumed so far. We have seen in Fig. 3 that $\mathrm{CN}$ has lower total average attenuation than MS, and thus with a SOA implementation of the interconnect the curves for $\mathrm{CN}$ in Fig. 7 should be lower than those for MS. The SOA implementation however is not free of problems, the most important being the backreflections and the backward ASE that tend to greatly enhance the ASE level, and the 


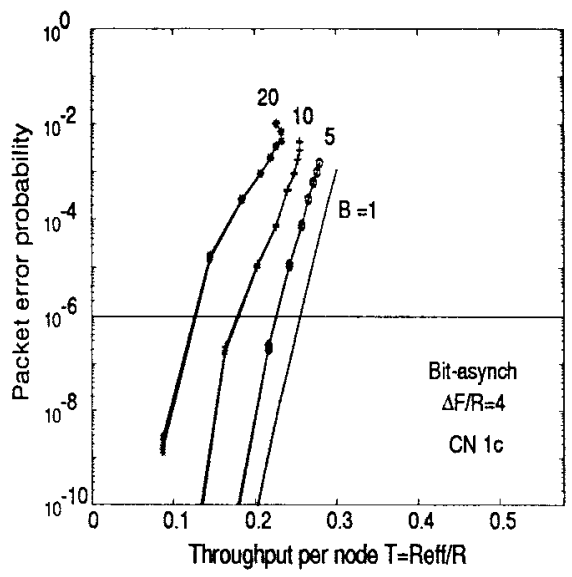

Fig. 9. PER versus throughput in $\mathrm{CN}$ with no buffers, same data as in Fig. 8(b), with bursty traffic, and average burst length $B=1,5,10$, and 20 cells.

decrease of the extinction ratio (the crosstalk factor $\alpha$ ) for large input powers [19]. Further investigation is needed to assess the potential benefits of an SOA-based design versus an EDFA-based design.

Comparisons have been performed for simplicity in uniform traffic, but the conclusions are of general validity, since PER degradations in nonuniform traffic are similar for the considered topologies and node schemes. A reduction in deflections and thus a throughput improvement is expected for more localized cell destinations, while deflections increase and throughput decreases with time-correlated traffic [3]. For example, Fig. 9 shows how PER degrades in $\mathrm{CN} 1 c$ when at each transmitter cell destinations are still uniform, but the destination is held constant for a burst of geometrically distributed size with mean $B=5,10$, and 20 cells. The case $B=1$ represents the uncorrelated case of Fig. 8(b). Throughput, link utilization and hop distribution curves have been obtained by simulation, since analytical formula are not available in this case. At high loads the degradation is less severe because of the burst fragmentation due to highpriority flow-through traffic at the access node. The throughput decrease for increasing load observed at the top of the curve for $B=20$ is an indication of network congestion, which is not observed for shorter burst lengths.

\section{CONCLUSIONS}

This work compares a weakly multihop centralized topology to the strongly multihop Manhattan Street topology for space-division transparent optical networks with deflection routing. The better connectivity provided by the centralized network allows less deflections, a lower link load, and a lower accumulated attenuation for the same carried traffic. Therefore $\mathrm{CN}$ has less crosstalk and potentially lower ASE levels than MS if suitably optimized gains in the interconnect are used. Centralized networks therefore can sustain larger throughputs at lower error rates than distributed, strongly multihop topologies. Also, less packet scrambling (delay jitter) can be obtained due to the reduced number of deflections. The price paid is an increase in the total number of elementary
$2 \times 2$ switches in the network, i.e., in hardware complexity at the central interconnect. Such price can be reduced if integrated interconnects can be realized.

A frequency sweeping technique has been shown to greatly alleviate the impact of coherent crosstalk in these singlewavelength networks, thereby allowing network operation with crosstalk factors of $-20 \mathrm{~dB}$ or worse. The centralized network, however, benefits less from the sweeping technique than the MS network, since the larger lumped losses in an implementation with EDFA's vanish the intrinsic lower losses of the $\mathrm{CN}$, which thus suffers from higher levels of spontaneous emission noise than MS. An implementation based on SOA's may give lower PER, but the issue needs further investigation.

Another way of decreasing both coherent crosstalk and deflections in all such networks is to deplete the optical transport layer by using faster transmitters/receivers (i.e., higher bit rates), thus supporting the same amount of traffic at lower loads. When this is done, less complicated, unbuffered structures $(1 c)$ turn out to be more attractive than more efficient, more lossy buffered structures $(2 c)$. This shifts the cost burden to speed-up the optical transmitter/receiver at the access node to allow major simplifications and cost reductions of the optical transport/switching part of the network.

\section{APPENDIX A}

This appendix derives the fundamental result (1). Consider a slotted network with $\mathrm{N}$ active nodes, i.e., nodes capable of injecting/absorbing cells. Let the active nodes have $k$ input links. We need not make any assumption on the number and degree of passive nodes, i.e., nodes dedicated only to routing. The network can be seen as a set of interconnected conveyor belts, each divided in bins, or slots, capable of carrying cells. Each belt moves at the speed of one slot per clock time. Routing nodes exchange slots from the incoming/outgoing conveyor belts according to their routing algorithm. Consider a "typical" slot moving around the network. It may be busy $(B)$ carrying a cell, or it may be idle $(I)$. The state of the slot then alternates between states $B$ and $I$ at each visit at an active node. Suppose the discrete-time evolution of the state of the slot, where time is measured at visits at active nodes, is an alternating renewal process [20]. On the average the slot remains busy for $H$ visits, and idle for $I$ visits. Thus the fraction of visits at which the slot is busy is

$$
u=\frac{H}{H+I} .
$$

Let $C \triangleq H+I=H / u$ be average life cycle of a slot. This is the number of hops the slot takes on average to deliver a cell. Hence, $1 / C$ is the average number of cells delivered by the slot at each hop, i.e., the probability that the slot has reached the cell's destination and delivers the cell.

Now, focus on an active node. At each clock it receives $k$ input slots, and each slot delivers on average $1 / C$ cells to it, so that the average number of absorbed cells per clock, i.e., 
the throughput per node, is

$$
T=\frac{k}{C}=\frac{k u}{H} \text {. }
$$

\section{APPENDIX B}

This Appendix finds the optimum gain values discussed in Section IV-B. Refer to Fig. B1. We are given $N$ lumped losses $L_{i}, i=1, \cdots, N$, and $N$ amplifiers with gains $G_{i}, i=$ $1, \cdots, N$, all with output saturation power $P_{\text {sat }}$. We start at point $P$ with power $P_{r x}$ and must end up in point $P^{\prime}$ with the same power (unity loop gain constraint). This requires

$$
\prod_{i=1}^{N} G_{i}=\prod_{i=1}^{N} L_{i} \triangleq L
$$

where $L$ is the total loss.

As in (4), the ASE power density at point P' can be written, using (B.1), as

$$
\begin{aligned}
N_{\mathrm{ase}}(1)=h \nu n_{s p}[ & \left(1-\frac{1}{L_{N}}\right) \\
& \left.+\sum_{i=0}^{N-2}\left(1-\frac{1}{L_{N-(i+1)}}\right) \prod_{j=N-i}^{N} \frac{G_{j}}{L_{j}}\right] .
\end{aligned}
$$

We want the gains $G_{i} \geq 1, i=1, \cdots, N$ that minimize $N_{\text {ase }}$ (1) in (B.2), subject to the saturation constraints

$$
\left\{\begin{aligned}
P_{o_{N-i}} & =\frac{P_{o_{N-(i-1)}}}{G_{N}(i-1)} L_{N-i} \leq P_{\mathrm{sat}}, \quad i=1, \cdots, N-1 \\
P_{o_{N}} & =P_{r x} L_{N} \leq P_{\mathrm{sat}}
\end{aligned}\right.
$$

where $P_{o_{i}}$ is the output power of the $i$ th amplifier. Such constraints can be combined, giving

$$
\prod_{j=N-i}^{N} \frac{G_{j}}{L_{j}} \geq \frac{L_{N-(i+1)}}{P_{\text {sat }} / P_{r x}} \quad i=0, \cdots, N-2 .
$$

Since gains and losses are positive quantities larger than $1, N_{\text {ase }}(1)$ in (B.2) is the sum of positive terms, which simultaneously reach their minimum when equality holds in (B.4).

Hence, the optimal gains are found iteratively from (B.4) (with the equality sign), starting from the Nth amplifier and proceeding leftwards. We get

$$
\left\{\begin{array}{l}
G_{N}=\frac{L_{N} L_{N-1}}{P_{\mathrm{sat}} / P_{r x}} \\
G_{j}=L_{j-1} \\
G_{1}=P_{\mathrm{sat}} / P_{r x} .
\end{array} \quad j=2, \cdots, N-1\right.
$$

Fig. 10 shows a diagram of the power levels in $\mathrm{dB}$ along the chain. We see that the optimization concept is to boost the power to the saturation value at the first amplifier, and then keep such saturation value at each amplifier output till the last one, that has to restore the initial power level $P_{r x}$. If some gain value in (B.5) cannot be obtained with a single amplifier, two or more cascaded amplifiers can be used.
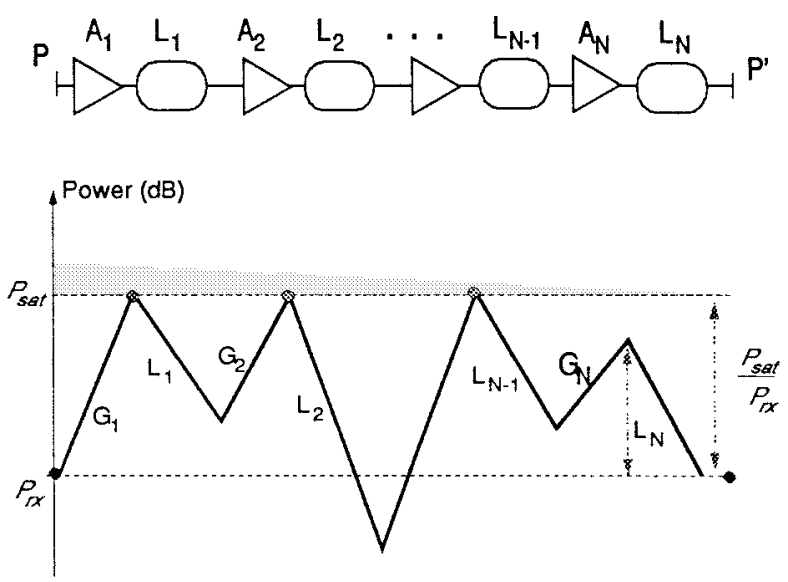

Fig. 10. The amplifier/loss chain to optimize and power variations along the chain.

With the optimal gains (B.5) we get from (B.2)

$$
\frac{N_{\text {ase }}(1)}{P_{r x}}=h \nu n_{s p}\left[\frac{L_{N}-1}{L_{N} P_{r x}}+\frac{\sum_{i=1}^{N-1}\left(L_{i}-1\right)}{P_{\text {sat }}}\right] \text {. }
$$

To minimize this, $P_{r x}$ should be as large as possible, i.e., from the second of (B.3), $P_{r x}=P_{\text {sat }} / L_{N}$, so that even the last amplifier works in saturation. For this case, $N_{\text {ase }}(1) / P_{r x}$ depends linearly on $\sum_{i=1}^{N} L_{i}$, subject to constraint (B.1), i.e., $\Pi_{i=1}^{N} L_{i}=L$ a constant. Thus, for given $N$ the optical SNR is maximum when the losses are equal, $L_{i}=L^{1 / N} \forall i$, for which we get a minimum value $N_{\text {ase }}(1) / P_{r x}=h \nu n_{s p}\left[N\left(L^{1 / N}-\right.\right.$ 1) $\left./ P_{\text {sat }}\right]$. This quantity decreases monotonically as $N \rightarrow \infty$ to the limit $h \nu n_{s p} \ln L / P_{\text {sat }}$, which is the asymptotic achievable minimum in the case of distributed amplification.

In our problem, we are given several lumped losses and a finite number of amplifiers, and from the above theory we know that we should try to place the amplifiers so as to make the interamplifier losses as even as possible. Also, the choice of $P_{r x}$ is not critical, as the first term in square brackets in (B.6) is, in our problem, much smaller than the second up to very low values of $P_{r x}$.

To complete the optimization problem, a particular case must still be considered. If for the Nth amplifier equation (B.4) gives a value of $G_{N}$ lower than one, then amplifier $N$ is not needed. We lump $L_{N-1}$ and $L_{N}$ in a single loss, and solve ther problem for $N-1$ amplifiers. This loss "lumping" is repeated till (B.4) gives a value $G_{N-k}>1$ for some $k \geq 0$. In other terms, one looks for $k \geq 0$ such that

$$
\frac{P_{\text {sat }}}{P_{r x}} \geq \prod_{j=N-k}^{N} L_{j} \triangleq L_{\hat{N}}
$$

and sets $G_{N-j}=1$ for $j=0, \cdots, k-1$. Then one uses the optimal solution given in (B.5) for the $\hat{N}=N-k$ remaining amplifiers. 


\section{APPENDIX C}

This appendix will derive the cross- and autocorrelation of the crosstalk components $x_{i}, x_{q}$ defined in (8). We have

$$
\begin{aligned}
R_{x_{i} x_{q}}(\tau)=\mathrm{E} & {\left[\alpha \sum _ { i = 1 } ^ { n _ { x t } } \sum _ { j = 1 } ^ { n _ { x t } } \mathrm { E } \left[m_{i}(t) m_{j}(t+\tau) \cos \Psi_{i} \cos \Psi_{j}\right.\right.} \\
& \left.\left.\cdot \cos \left(\Delta \omega_{i} t+\Phi_{i}\right) \sin \left(\Delta \omega_{j}(t+\tau)+\Phi_{j}\right) / n_{x t}\right]\right]
\end{aligned}
$$

where the outer expectation on the right hand-side (RHS) is taken with respect to $n_{x t}$. All terms with $i \neq j$ average to zero because $\mathrm{E}\left[\cos \Psi_{i}\right]=0$, being $\Psi_{i}$ uniform and independent of $n_{x t}$. Considering that $\mathrm{E}\left[m_{i}(t) m_{i}(t+\tau)\right] \triangleq R_{m}(\tau)$ for all $i$ 's, and that $\mathrm{E}\left[\cos ^{2}\left(\Psi_{i}\right)\right]=1 / 2$, we have

$$
\begin{aligned}
R_{x_{i} x_{q}}(\tau)=\mathrm{E} & {\left[\alpha \sum_{i=1}^{n_{x t}} R_{m}(\tau) \frac{1}{2} \mathrm{E}\right.} \\
& \left.\cdot\left[\frac{\sin \Delta \omega_{i} \tau+\sin \left(\Delta \omega_{i}(2 t+\tau)+2 \Psi_{i}\right)}{2}\right]\right]=0
\end{aligned}
$$

because $\mathrm{E}\left[\sin \Delta \omega_{i} \tau\right]=0 \forall i$, since the probability density function (pdf) of $\Delta \omega_{i}$ is symmetric around zero, and $\mathrm{E}\left[\sin \left(\Delta \omega_{i}(2 t+\tau)+2 \Psi_{i}\right)\right]=0$ because $2 \Psi_{i}$ is uniform.

Analogously

$$
\begin{aligned}
R_{x_{i}}(\tau)=\mathrm{E}\left[\alpha \sum _ { i = 1 } ^ { n _ { x t } } \sum _ { j = 1 } ^ { n _ { x t } } \mathrm { E } \left[m_{i}(t) m_{j}(t+\tau) \cos \Psi_{i} \cos \Psi_{j}\right.\right. \\
\left.\left.\cdot \cos \left(\Delta \omega_{i} t+\Phi_{i}\right) \cos \left(\Delta \omega_{j}(t+\tau)+\Phi_{j}\right) / n_{x t}\right]\right] \\
=\mathrm{E}\left[n_{x t}\right] \alpha R_{m}(\tau) \frac{1}{2} \sum_{i} \mathrm{E}\left[\frac{1}{2} \cos \Delta \omega_{i} \tau\right]
\end{aligned}
$$

This establishes (15), by noting that $\mathrm{E}\left[e^{j \Delta \omega_{i} \tau}\right]=$ $\mathrm{E}\left[\cos \Delta \omega_{i} \tau\right]=\operatorname{sinc}^{2}(\Delta F \tau)$, being this the characteristic function of the RV $\Delta \omega_{i}$, i.e., the Fourier transform of its triangular-shaped pdf $(1 / \pi \Delta F) \Lambda(\Delta \omega / 2 \pi \Delta F)$.

1) Evaluation of $E\left[n_{x t}\right]$ : Let $C$ be the number of hops added by each deflection. For $\mathrm{CN} C=1$, while for MS $C=4$ [8]. Let $H_{\min }$ be the value of the average number of hops at loads approaching zero, when no deflections occur (see Fig. 2). Given a path of $n$ hops, we approximate the number of deflections along the path as $n_{d}=\lfloor\max (n-$ $\left.\left.H_{\min }, 0\right) / C\right\rfloor$. Since a deflection implies that the tagged cell meets $x$ competing cells at a routing element $(x=1$ for $1 c$ and $x=2$ for $2 c$ ), on a path of $n$ hops there are at least $x \cdot n_{d}$ crosstalk interferers. Let $N_{x}$ be the number of points along the path where crosstalk may arise (crosstalk points, see Table I). If we assume that at the $N_{x}-x \cdot n_{d}$ points where deflection does not occur an interferer is present with probability $u$ (the link load defined in Section II), then $\mathrm{E}\left[n_{x t}\right]=x \cdot n_{d}+u\left(N_{x}-\left(x \cdot n_{d}\right)\right)$.

\section{ACKNOWLEDGMENT}

The author would like to acknowledge Dr. P. Adorni for stimulating discussions. This work was performed within a collaboration between Università di Parma and CSELT.

\section{REFERENCES}

[1] E. L. Goldstein and L. Eskildsen, "Scaling limitations in transparent optical networks due to low-level crosstalk," IEEE Photon. Technol. Lett., vol. 7, pp. 93-94, Jan. 1995.

[2] P. Baran, "On distributed communications networks," IEEE Trans. Commun. Syst., vol. COM-12, pp. 1-9, Mar. 1964

[3] F. Forghieri, A. Bononi, and P. R. Prucnal, "Analysis and comparison of hot-potato and single-buffer deflection routing in very high bit rate optical mesh networks," IEEE Trans. Commun., vol. 43, pp. 88-98, Jan. 1995.

[4] C. Rose, "Mean internodal distance in regular and random multihop networks," IEEE Trans. Commun., vol. 40, pp. 1310-1318, Aug. 1992.

[5] A. G. Greenberg, and B. Hajek, "Deflection routing in hypercube networks," IEEE Trans. Commun., vol. 40, pp. 1070-1081, June 1992.

[6] W. D. Hillis, The Connection Machine. Cambridge, MA: MIT Press, 1985.

[7] D. J. Blumenthal, K. Y. Chen, J. Ma, R. J. Feuerstein, and J. R. Sauer, "Demonstration of a deflection routing $2 \times 2$ photonic switch for computer interconnects," IEEE Photon. Technol. Lett., vol. 4, pp. 169-173, Feb. 1992.

[8] N. F. Maxemchuk, "Comparison of deflection and store-and-forward techniques in the Manhattan Street and Shuffle-Exchange networks," in Proc. IEEE INFOCOM '89, Apr. 1989, pp. 800-809.

[9] A. Bononi, "Space-division optical star networks with deflection routing," in Proc. 1996 Conf. Inform. Sci. Syst., Princeton, NJ, vol. 2, pp. 1113-1118, Mar. 1996.

[10] O. K. Tonguz and L. G. Kazovsky, "Theory of direct-detection lightwave receivers using optical amplifiers," J. Lightwave Technol., vol. 9, pp. 174-181, Feb. 1991.

[11] O. K. Tonguz and K. A. Falcone, "Fiber-optic interconnection of local area networks: Physical limitations of topologies," J. Lightwave Technol., vol. 11, pp. 1040-1052, May/June 1993.

[12] N. A. Olsson, "Lightwave systems with optical amplifiers," J. Lightwave Technol., vol. 7, pp. 1071-1082, July 1989.

[13] P. A. Humblet and M. Azizoglu, "On the bit error rate of lightwave systems with optical amplifiers," J. Lightwave Technol., vol. 9, pp. 1576-1582, Nov. 1991.

[14] L. Moura, N. Karafolas, P. Lane, A. Hill, and J. O'Reilly, "Modeling of interferometric crosstalk in optical networks," in Proc. IEEE GLOBECOM '96, London, U.K., Nov. 1996, pp. 333-337.

[15] A. Papoulis, Probability, Random Variables and Stochastic Processes, 2nd ed. New York: McGraw-Hill, 1984, p. 321.

[16] Probability, Random Variables and Stochastic Processes, 2nd ed. New York: McGraw-Hill, 1984, pp. 228-230.

[17] M. Abramowitz and I. A. Stegun, Handbook of Mathemtical Functions New York: Dover, 1970, p. 231.

[18] R. R. Ramaswami and P. A. Humblet, "Amplifier induced crosstalk in multichannel optical networks," J. Lightwave Technol., vol. 8, pp. 1882-1896, Dec. 1990.

[19] E. Almström, C. P. Larsen, L. Gillner, W. H. van Berlo, M. Gustavsson, and E. Berglind, "Experimental and analytical evaluation of packaged $4 \times 4$ InGaAsP/InP semiconductor optical amplifier gate switch matrices for optical networks," J. Lightwave Technol., vol. 14, pp. 996-1004, June 1996.

[20] J. M. Ross, Stochastic Processes. New York: Wiley, 1983, pp. 55-99.

Alberto Bononi (M'90) received the "Laurea in Ingegneria Elettronica" cum laude from the University of Pisa, Pisa, Italy, in 1988 and the M.A. and Ph.D degrees in electrical engineering from Princeton University, Princeton, NJ, in 1992 and 1994, respectively.

From 1994 to 1995, he was an Assistant Professor in the Electrical and Computer Engineering Department at the State University of New York (SUNY) Buffalo. Since 1996, he has been an Assistant Professor at the Università di Parma, Italy. His current research interests include system design and performance issues in fast packet switching and high-speed all-optical networks, and nonlinear fiber transmission for WDM systems. 